\title{
Physiological Responses to Maximal Exercise on Arm Cranking and Wheelchair Ergometer with Paraplegics
}

\author{
G. Martel, MSc, ${ }^{1}$ L. Noreau, PhD, ${ }^{1}$ J. Jobin, PhD $^{2}$ \\ ${ }^{I}$ Adapted Physical Activity Evaluation Laboratory, Centre François-Charon, 525 Blvd \\ Hamel, Quebec City, G1M 2S8, ${ }^{2}$ Professor, School of Nursing, Laval University, \\ Quebec City, G1K 7P4, Canada.
}

\section{Summary}

This study describes the responses of 20 paraplegic athletes (mean age: $26.8 \pm 1.6$ years) to a continuous incremental workload test until exhaustion on an arm cranking ergometer $(A C E)$ and on a wheelchair erogmeter (WCE). Both ergometers used the same electromagnetic braking device allowing a fair comparison between results. Tests were conducted at a 24 hour interval at the same time of the day. Oxygen uptake $\left(\mathrm{VO}_{2}\right)$, heart rate $(H R)$, workload $(W)$, blood pressure $(B P)$, Borg index, and mechanical efficiency $(M E)$ were measured at every minute during the effort and the cool down periods of both tests. The purpose of this study was to analyse the different responses obtained on ACE and on WCE during maximal effort by paraplegics, and also to determine which ergometer permits the higher $M E$. Results indicate that paraplegics reached the same max $H R$ on $A C E$ and on WCE (97\% of the predicted max HR). The lack of significant difference $(p<0.05)$ between $A C E$ and $W C E$ in terms of maximal values of $V \mathrm{O}_{2}, V E$ and $H R$ suggests that the subjects reached their maximal capacity on each test regardless of the type of ergometer. Nevertheless, W max (in Watts) was $26 \%$ higher on ACE than on WCE. Maximal ME values were respectively $16 \%$ and $11 \cdot 6 \%$ on ACE and WCE. Results suggest that ergometers and protocol used in this study are appropriate to measure physiological responses of paraplegic athletes during arm cranking and wheelchair exercise without excessive or early arm fatigue.

Key words: Paraplegics; Exercise testing; Wheelchairs; Arm cranking.

Arm ergometry has been the subject of many studies during the past decade in order to deal with the special needs of people with disabilities (Asayama et al., 1985; Bezucha et al., 1982; Bobbert, 1960; Claremont, 1985; Cooney, 1986; Gass et al., 1980). Vigorous efforts have been made to develop proper models of physical fitness assessment for special populations. 
Exhaustive literature reviews on exercise training and arm ergometry for spinal cord injury (SCI) persons have been published (Dreisinger and Londeree, 1982; Glaser, 1985; Hoffman, 1986; Sawka, 1986; Shephard, 1988). Among the many topics concerning exercise testing and training, is the description of exercise protocols used for SCI persons to elicit physiological modifications. Recent studies on the working capacity of paraplegics have shown significant differences in physiological variables when exercise is produced by different muscle groups or by the same muscles in different patterns of movements (Gass and Camp, 1984; Glaser et al., 1980; Horvat et al., 1986).

Specificity of exercise such as was described for able-bodied persons (Bouchard et al., 1979) seems to be a major concern also with SCI people. Data gathered during exercise testing using an adapted ergometer could be used to elaborate an optimal training program early in the rehabilitation process of paraplegic patients.

Arm cranking ergometer (ACE) and wheelchair ergometers (WCE) are amongst the most commonly used devices for the cardiorespiratory assessment of SCI persons. Many parameters such as oxygen uptake $\left(\mathrm{VO}_{2}\right)$, ventilation $(\mathrm{Ve})$, blood pressure (BP), heart rate (HR), and mechanical efficiency (ME) have been measured in paraplegics during exercise. However, most of the studies have a significant disparity in subject selection, sampling methods, measuring devices, and protocols. This led to an absence of homogeneity or a common denominator which did not allow researchers to properly compare their results in terms of physiological modifications induced by exercise, or in terms of an adequate measurement of movement mechanical efficiency.

The purpose of the present study is to compare physiological adjustments to arm cranking and wheelchair exercise in a group of 20 paraplegics using an ACE and a WCE during maximal exercise tests to exhaustion. Since both ergometers used the same electromagnetic braking system, the use of the same continuous protocol has been made possible. Furthermore, this study will analyse the differences of mechanical efficiency between ACE and WCE.

\section{Methods}

\section{Subjects}

Informed consent was obtained from 20 volunteer male paraplegics (mean age $26.8 \pm 1.6$ years) prior to participation in this study. A medical examination was performed on all subjects before the first day of testing. Security standards currently recognised by the American College of Sports Medicine, (1986) were applied during each session of testing. Most of the subjects were involved in wheelchair sports (Wheelchair racing, basketball, handball). The physical fitness of the subjects was compatible with the requirements of a maximal graded exercise test. Table I summarises the characteristics of each participant. We have also classified the subjects according to their level of injury as proposed by the Internal Stoke Mandeville Games Federation (ISMGF) (McCann, 1984). (See Table II).

\section{Instrumentation}

The construction of the ACE and the WCE was made possible by a modification of 
Table I Characteristics of the subjects

\begin{tabular}{|c|c|c|c|c|c|}
\hline Subject & $\begin{array}{l}\text { Level of } \\
\text { injury }\end{array}$ & Classification & Age & $\begin{array}{c}\text { Training } \\
\text { Hours/week }\end{array}$ & $\begin{array}{l}\text { No. of years in } \\
\text { wheelchair }\end{array}$ \\
\hline 1 & T6-T7 & 3 & 28 & 15 & 10 \\
\hline 2 & T11-T12 & 4 & 27 & 3 & 3 \\
\hline 3 & T6-T7 & 3 & 24 & 10 & 24 \\
\hline 4 & T11-T12 & 4 & 16 & 10 & 13 \\
\hline 5 & Т8-Т9 & 3 & 34 & 1 & 3 \\
\hline 6 & T11-T12 & 4 & 22 & 6 & 4 \\
\hline 7 & T3-T4 & 2 & 28 & 2 & 9 \\
\hline 8 & T11-T12 & 4 & 24 & 4 & 7 \\
\hline 9 & T6-T7 & 3 & 19 & 6 & 2 \\
\hline 10 & T7-T8 & 3 & 21 & 2 & 6 \\
\hline 11 & T6-T7 & 3 & 24 & 3 & 6 \\
\hline 12 & $\mathrm{~T} 4-\mathrm{T} 5$ & 2 & 25 & 4 & 5 \\
\hline 13 & T12-L1 & 4 & 49 & 2 & 38 \\
\hline 14 & T10-T11 & 3 & 35 & 2 & 3 \\
\hline 15 & T10-T11 & 3 & 28 & 3 & 21 \\
\hline 16 & T4-T5 & 2 & 32 & 12 & 9 \\
\hline 17 & T11-T12 & 4 & 32 & 8 & 27 \\
\hline 18 & L4-L5 & 5 & 25 & 5 & 7 \\
\hline 19 & T11-T12 & 4 & 25 & 2 & 3 \\
\hline 20 & $\mathrm{~T} 12-\mathrm{L} 1$ & 4 & 28 & 6 & 4 \\
\hline
\end{tabular}

Table II Classification of subjects according to ISMGF

\begin{tabular}{lcc}
\hline Level of injury & Class & No. of subject: \\
\hline C6-C8 & 1 & 0 \\
T1-T5 & 2 & 3 \\
T6-T10 & 3 & 9 \\
T11-L3 & 4 & 7 \\
L4-S2 & 5 & 1 \\
Minimal deficit & 6 & 0 \\
\hline
\end{tabular}

a 'Lode Corival 300' cycle ergometer braking device, by using the same electromagnetic braking system for both ergometers. The workload (W) (in watts) was similar at each corresponding stage. Moreover, this system has the advantage of producing the same inertia found during wheelchair propelling on the road. The accuracy of the workload system is \pm 0.5 watt for a speed ranging between 40 and 80 RPM.

The minimum and the maximum $\mathrm{W}$ offered by this system are respectively 0 and 300 watts. The elevation and the inclination of the seat, the foot rest, and the wheels of the WCE are fully adjustable to fit the specifications and ensure the comfort of every subject. In order to prevent major BP falls, especially caused by a lack of sympathetic tone in the muscles below the level of the spinal cord lesion, the method of legs positioning currently employed by wheelchair competitors during maraton racing was encouraged.

During the ACE testing, each subject had to be seated in a conventional table chair with their legs and their hips fixed with belts for optimal stability. The axle of 
the ACE was adjusted at the shoulders height of each participant. A similar range of speed had to be maintained on the ACE and on the WCE during both exercise tests.

A metabolic measurement cart 'Morgan Magna 88' coupled with an Apple IIe micro computer was used to collect physiological data every 30 seconds during the test. The exhaled pulmonary gases were continuously analysed for $\mathrm{O}_{2}$ and $\mathrm{CO}_{2}$ content. The exercise electrocardiogram was recorded with a Hewlett Packard 4700A Cardiograph. The Borg scale of perceived exertion was used to determine the level of fatigue reached by the subjects in the middle and at the end of each test.

\section{Protocol}

Prior to the first exercise test, measures of the height, weight, BP, resting HR, and a 12 lead electrocardiogram were collected. Both tests were conducted at least 24 hours apart and at the same time of day. Criteria to stop the test were those specified by the American College of Sports Medicine, the reaching of the maximal predicted HR (220-age), a score of 17 on the Borg scale, or the exhaustion of the subject.

Each participant was asked to perform a maximal continuous exercise test to exhaustion starting with a 2 minute warm-up period at an initial $W$ of 5 watts. This warm-up period was also meant to allow each subject to be familiar with the system and to adjust to a comfortable speed. An increment of 10 watts was made every minute until the end of the test. The HR was monitored on electrocardiogram using a CM5 lead. BP data was taken manually every 2 minutes during the arm cranking test only.

Regarding the level of the HR during both tests, the Borg index was collected at $50 \%$ of the estimated maximal $\mathrm{HR}$ and during the last minute of each test. The rating of perceived exertion was collected for central $\left(\mathrm{HR}, \mathrm{Ve}, \mathrm{VO}_{2}\right)$ and local (arm and shoulder muscles) perception of effort. An active cool down period of 6 minutes was followed by 5 minutes of rest in a sitting position during which the ECG was monitored. Gross ME calculation was made in accord with the formula used by Gaesser and Brooks (1975) and transformed in kilo-joules by Coutts et al. (1983).

$$
\text { Gross } \mathrm{ME}=\frac{\mathrm{W} \star 60 \star 10^{-3}}{\mathrm{VO}^{2} \star 21 \mathrm{kj} \cdot \mathrm{min}^{-1}}{ }^{\star} 100
$$

Statistical analysis

Statistical program SAS and Statview $512^{+}$were used for data processing. Statistical analysis included descriptive data (means, standard deviation), Pearson correlations, t-test, analysis of variance (ANOVA) between classes, and regression. Significance for all statistical analysis was fixed at a 05 confidence level $(\mathrm{p}<0.05)$.

\section{Results}

\section{Physiological response}

The 20 paraplegics performed a maximal graded exercise test on ACE and on WCE 
Table III Results of maximal values from exercise testing on ACE and WCE

\begin{tabular}{|c|c|c|c|c|c|}
\hline & \multicolumn{2}{|c|}{ ACE } & \multicolumn{2}{|c|}{ WCE } & \multirow[b]{2}{*}{$\mathrm{p}$} \\
\hline & $\mathrm{X} \pm \mathrm{SD}$ & (Spread) & $\mathrm{X} \pm \mathrm{SD}$ & (Spread) & \\
\hline HR max (bpm) & $190 \pm 15$ & $(154-213)$ & $190 \pm 13$ & $(160-210)$ & NS \\
\hline W max (Watt) & $97 \pm 25$ & $(70-150)$ & $74 \pm 19$ & $(40-110)$ & $\delta$ \\
\hline VE $\max (\mathrm{L} / \mathrm{min})$ & $897 \cdot 4 \pm 25 \cdot 0$ & $(45 \cdot 9-125 \cdot 0)$ & $95 \cdot 7 \pm 24 \cdot 8$ & $(60 \cdot 9-148 \cdot 1)$ & NS \\
\hline $\mathrm{VO}_{2}$ peak $(\mathrm{L} / \mathrm{min})$ & $1 \cdot 88 \pm \cdot 62$ & $(1 \cdot 15-3 \cdot 24)$ & $1.90 \pm .63$ & $(1 \cdot 00-2 \cdot 89)$ & NS \\
\hline $\mathrm{ME}(\%)$ & $16 \cdot 2 \pm 2 \cdot 0$ & $(14 \cdot 1-21 \cdot 1)$ & $11 \cdot 6 \pm 1.6$ & $(8 \cdot 6-14 \cdot 3)$ & $\delta$ \\
\hline
\end{tabular}

NS: no significant difference.

f: $\mathrm{p}<\cdot 05$.

in accordance with the criteria established at the beginning of this study. Mean speed chosen by the participants was $75.0 \pm 4.0 \mathrm{rpm}$ on ACE and on WCE. None of the subjects had ECG abnormalities during both tests. Only 1 paraplegic (T4-T5) had a fall in systolic pressure during the cool down period.

Table III summarises the results of both maximal exercise tests. No difference was observed between tests on ACE and WCE for maximal values of heart rate (HR $\max$ ), ventilation Ve max, and oxygen uptake $\mathrm{VO}_{2}$ peak. The average maximal $\mathrm{HR}$ of the subjects during the tests equalled $97 \%$ of the estimated maximal $\mathrm{HR}$ values (220-age). A significant difference was observed in maximal W between ACE and WCE procedures. The maximal $\mathrm{W}$ was $24.6 \pm 10 \cdot 8 \%$ higher on ACE than on WCE. A strong positive association was found between ACE and WCE for peak $\mathrm{VO}_{2}(\mathrm{r}=\cdot 84)$, Ve $\max (\mathrm{r}=\cdot 84), \mathrm{HR} \max (\mathrm{r}=\cdot 85)$, and $\mathrm{W} \max (\mathrm{r}=\cdot 90)$.

\section{Perceived exertion}

No significant association $(p>\cdot 05)$ was found in central and local perceived exertion at sub-maximal exercise $(50 \% \mathrm{HR})$. At maximal exercise, a moderate association $(r=\cdot 65)$ was observed for local perceived exertion between ACE and

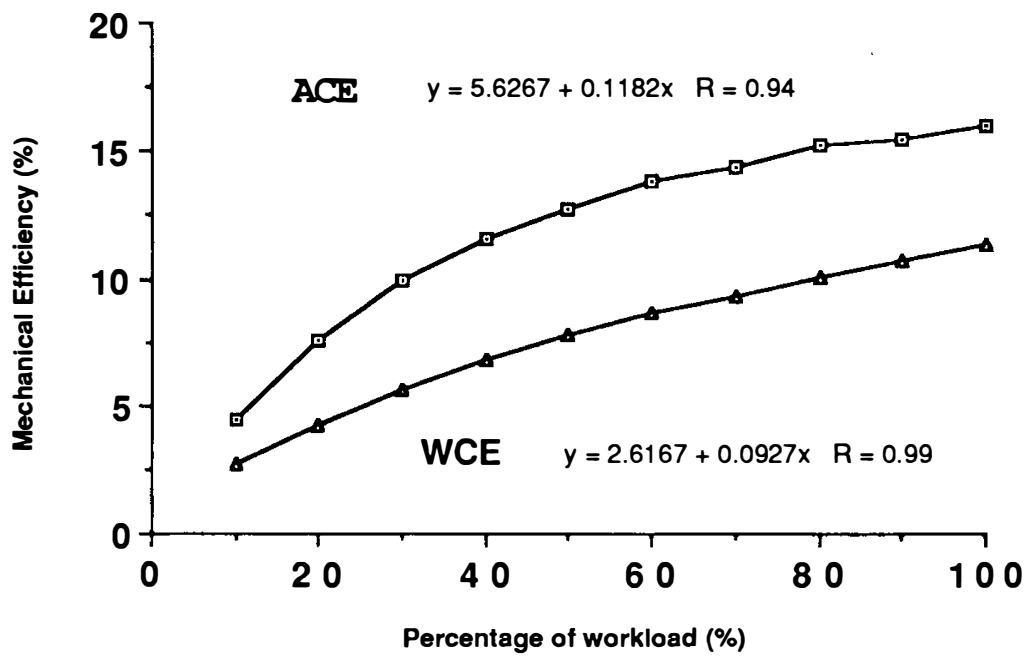

Figure Evolution of the ME curves on ACE and on WCE. 
WCE, but no significant association was found for central perceived exertion. Likewise, there was no significant association between central and local perceived exertion in both procedures when analysed separately.

\section{Mechanical efficiency}

Gross mechanical efficiency computed for maximal exercise showed a significant difference between ACE and WCE $(16 \cdot 2$ vs $11 \cdot 6 \% \mathrm{p}<\cdot 05)$. The figure illustrates the evolution of the curves of $\mathrm{ME}$ in relation to the $\mathrm{W}$ percentage of both ergometers.

For all variables previously mentioned, ANOVA procedure was performed in order to determine the effect of ISMGF classes on these variables. This analysis failed to indicate significant differences between ISMGF classes (2, 3 and 4$)$ in ACE and WCE.

\section{Discussion}

\section{Physiological adjustments}

Many studies on exercise testing have focused on the measure of movement specificity with different populations performing different tasks (Bobbert, 1960; Bergh et al., 1976; Bouchard et al., 1979; Sawka et al., 1982; Eston et al., 1986). Assessing movement specificity with SCI persons may lead researchers towards a better understanding of their response to different modes of exercise. This can also be worthwhile by involving patients in a more active participation in their physical rehabilitation.

Comparison of the physiological responses obtained during exercise testing on ACE and on WCE has been made in some studies (Gass and Camp, 1984, Wicks et al., 1978, Sawka et al., 1980; Glaser et al., 1980; Smith et al., 1983, Wicks et al., 1983). Only a small number of these authors have assessed a group of paraplegics during an exercise test to exhaustion by utilising the same testing protocol on both ergometers. In the literature, utilisation of different mechanical systems for ACE or WCE might partly explain the large variety of protocols used in exercise testing.

Furthermore, in many studies, data concerning stress testing using these two ergometers are different depending on the type of protocol used (Kofsky et al., 1983; Gass and Camp 1984; Dotan and Bar-Or 1983; William et al., 1983), the disability of subjects (Horvat et al., 1986; Kavanagh and Shephard 1973; Wahren and Bygdeman 1971, Wilde et al., 1981), and the characteristics of ergometers (Bergh et al., 1976; Nagle et al., 1984; Lundberg, 1980).

In the present study, emphasis is laid on the similarity of functioning of both types of ergometers. The utilisation of the same electromagnetic braking device for the ACE and the WCE in the present study has permitted us to properly analyse data in paraplegic subjects when performing incremental workload tests to exhaustion with a fair accuracy using either ergometer.

Results presented in this study show no significant difference between maximal values of $\mathrm{VO}_{2}, \mathrm{HR}$, and Ve measured on ACE and on WCE. These results are in agreement with those of Glaser et al. (1980) and Wicks et al. (1983) for $\mathrm{VO}_{2}$ peak and Vemax values. High correlations indicate that responses to exercise between 
ACE and WCE are very consistent. Usually wheelchair exercise elicits a lower HR max than arm cranking exercise (Sawka et al.,1986). Our results show that both ergometers produce similar HR max. These results can be partially explained by the fact that our subjects were familiar with training in wheelchair sports.

In a review on the difference between lower and upper body exercise, Franklin, (1985) reveals that maximal heart rate elicited by maximal exercise on a treadmill or on a stationary bicycle is 3 to $23 \mathrm{bpm}$ higher than the HR reached by maximal upper body testing. Total muscle mass involved in the movement and the specificity of training seems to be at the origin of these disparities (Franklin, 1985; Bergh et al., 1976).

Interestingly, our subjects reached $97 \%$ of the predicted HR max, corresponding to only $3 \mathrm{bpm}$ lower than the predicted HR max (220-age) for each subject, and some of these reached a value higher than 200 beats $/$ min $(100-110 \%$ of predicted HR max).

At maximal exercise, everyone has to sustain an adequate cardiac output in order to reach their $\mathrm{VO}_{2}$ max. In SCI persons, the components of cardiac output (HR and stroke volume) are modified (Bruin et al., 1984; Hjeltnes, 1980). The loss of central sympathetic tone below the injury level inhibits vasoconstriction in inactive tissues causing the mean blood pressure to diminish, causing a blood pooling in the lower limb (Shephard, 1988). Venous return is decreased. This leads to a lower end-diastolic volume and stroke volume. The static component in arm cranking and wheelchair exercise may also have an adverse effect on cardiac haemodynamics in increasing the afterload (Sawka, 1986). Thus, we can assume that a proper cardiac output cannot be sustained without a compensatory increase in HR. Such a response to maximal exercise was likely to appear, considering the fitness level and training regimen of the subjects.

Maximal W measured at exhaustion was $24 \cdot 6 \pm 10 \cdot 8 \%$ higher on ACE than on WCE. Individually, each paraplegic of this study reached higher W values on ACE even though our subjects were accustomed to training on a wheelchair. In a similar research, Glaser et al. (1980) found a difference of $36 \%$ for $W \max$ values at the end of testing in favour of ACE.

The physiological stress imposed by the synchronous movement pattern on WCE compared with a continuous movement performed on ACE, may elicit a greater static component (Davis et al., 1981; Glaser, 1985), producing a Valsalva manoeuvre at each thrust (Mostardi and Gandee, 1981), which can momentarily decrease blood flow to the working muscles. This type of movement is more likely to allow an accumulation of metabolic by-products in the blood and may reduce the duration of the exercise. In another literature review, Dreisinger et al. (1982) consider that for the same $\mathrm{W}$, cardiovascular stress is more important when using conventional wheelchair than arm cranking.

\section{Perceived exertion}

In a study comparing perceived effort on an ACE and a stationary bicycle during a 60 minute exercise testing, Pandolf et al. (1984) found, for the same workload, higher RPE values on ACE suggesting that muscle mass could be an important factor in the determination of perceived fatigue. Low correlations found in local and central perceived exertion betwen ACE and WCE exercise in the present 
study, tend to suggest that both wheelchair and arm cranking exercise, are very specific exercises and could probably be unrelated in terms of perceived exertion.

However, several factors such as mechanics of the movement (synchronous vs asynchronous pattern), habits of wheelchair sports, and conditioning level could influence the RPE in upper-body exercise. Nonetheless, it is obvious that other non-controlled factors in the present study would have influenced RPE in upper body exercise.

\section{Mechanical efficiency}

Calculation of gross $\mathrm{ME}$ provides valuable information on the relationship between the energy expenditure and the peak work load. Comparing two types of ergometers with ME data as criteria can be advantageous in order to choose the proper ergometer for exercise testing or training.

The results of the present research support those found by several others (Smith et al., 1983; Glaser et al., 1980) and show a significant difference between maximal $M E$ values reached by the twenty paraplegics. This indicates that an exercise test on ACE can be more efficient in terms of $\mathrm{VO}_{2}$ and $\mathrm{HR}$ for the same amount of work on WCE, suggesting that arm cranking exercise is a more efficient movement for locomotion in paraplegics. Many factors can be considered when analysing the differences observed between gross ME on ACE and on WCE. The muscles involved in both types of movement are different even if their total mass is similar, as the present data on $\mathrm{VO}_{2}$ max tends to demonstrate. Davis et al. (1981) suggest that muscles involved in the stabilisation of the trunk during exercise on WCE may take an important part of the energy expended during the test.

It is interesting to mention that the total time spent to bring hands back to the starting point of the double push action on WCE can produce a significant speed reduction of the wheels for a while and might require from the subject a surplus of energy when initiating each thrust. As the workload is getting heavier at the end of the test, the braking effect of the system increases gradually, and the slowing down rate may become more perceptible for subjects.

The type of muscular contraction seems to give different responses during both movements. The isometric muscular contraction to initiate the action, completed by an eccentric arm movement on WCE handrims, may induce different physiological modifications when compared with those recorded on ACE. Hanse and Maggio (1960) have measured a higher sympathetic tone during an isometric contraction compared with an isotonic movement: it produces a faster elevation of HR during the exercise. Studying also the physiological effects of an isometric contraction for an upper body work, Sawka et al. (1983) found a higher concentration of lactic acid in the blood and a higher intra-muscular BP.

Factors mention above may induce a larger utilisation of anaerobic pathways in wheelchair exercise, producing a considerable accumulation of metabolic byproducts. They might contribute to explain the smaller $\mathrm{ME}$ values recorded on the WCE.

\section{Conclusion}

The results of the present study suggests that the protocol and ergometers used in 
this study are appropriate to measure physiological responses of paraplegic athletes during an incremental arm cranking or wheelchair exercise without excessive or early arm fatigue. The also indicate, in terms of energy saving, that $\mathrm{ME}$ values favoured working on ACE rather than on WCE. Nonetheless, considering the concept of specificity of exercise and that both modes of exercise tend to elicit similar $\mathrm{VO}_{2}$ peak and $\mathrm{HR}$ max, wheelchair exercise is more appropriate it improves the capability in using a manual wheelchair (Glaser, 1985). Furthermore, a wheelchair is the most popular locomotion device in SCI persons and, it seems appropriate to encourage physical training with such a system.

The paucity of information found in the literature about specificity of exercise testing with the SCI population keeps doors wide open in this field of research. To facilitate physical rehabilitation, much research dealing specifically with the spinal cord injured persons responses to exercise should be encouraged in the future.

\section{References}

American College of Sport Medicine. 1986 Guidelines for Exercise Testing and Prescription, 3rd Edn. Lea \& Febiger, Philadelphia.

asayama K, Nakamura Y, Ogata H, Hatada K, Okuma H, Deguchi Y 1985 Physical fitness of paraplegics in full wheelchair marathon racing. Paraplegia 23:277-287.

BERGH U, KANSTRUP IL, EKBLOM B 1976 Maximal oxygen uptake during exercise with various combinations of arm and leg work. Fournal of Applied Physiology 41(2):191-196.

BezUCha GR, LENSER MC, HaNSON PG, NAGLE FG 1982 Comparison of hemodynamic responses to static and dynamic exercise. J Applied Physiol: Respiratory Environmental Exercise Physiology 53(6):1589-1593.

BOBBERT AC 1960 Physiological comparison of three types of ergometry. Fournal of Applied Physiology 15(6):1007-1014.

BouChaRd C, Godbout P, Mondor JC, Leblanc C 1979 Specificity of maximal aerobic power. Fournal Applied Physiology 40:85-93.

BRUIN MI, BINKHORST RA 1984 Cardiac output of paraplegics during exercise. International fournal of Sports Medicine 5:175-176.

ClaREMONT AD, MAKSUD MG 1985 A model treadmill adaptation for wheelchair ergometry. Canadian Fournal of Applied Sports Science 10(4):178-181.

COONEY MM, WALKER JB 1986 Hydraulic resistance exercise benefits cardiovascular fitness of spinal cord injured. Medicine and Science in Sport and Exercise 18(5):522-525.

CoutTs K, RHODEs EC, MCKENZIE DC 1983 Maximal exercise responses of tetraplegics and paraplegics. Fournal of Applied Physiology 55(2):479-482.

DAVIS GM, SHEPHARD RJ, JACKSON RW 1981 Cardio-respiratory fitness and muscular strength in the lower limb disabled. Canadian Fournal of Applied Sports Science 6(4):159-165.

DOTAN R, BAR-OR O 1983 Load optimisation for the Wingate anaerobic test. European fournal of Applied Physiology 51:409-417.

DREISINGER TE, LONDEREE BR 1982 Wheelchair exercise: a review. Paraplegia 20:20-34.

ESTON RG 1986 Responses to arm and leg ergometry. British fournal of Sports Medicine 20(1):4-6.

GAESSER GA, BROOKS GA 1975 Muscular efficiency during steady-rate exercise: effects of speed and work rate. Fournal Applied Physiology 38(6):1132-1139.

Gass GC, Watson J, CAMP EM, Court HJ, MCPherson LM 1980 The effects of physical training on high level spinal cord patients. Scandinavian fournal of Rehabilitation Medicine 12:61-65.

GASS GC, CAMP EM 1984 The maximum physiological responses during incremental wheelchair and arm cranking exercise in male paraplegics. Medical Science in Sports and Exercise 16(4):355-359.

GLASER R 1985 Exercise and locomotion for the spinal cord injured. Exercise and Sport Science Reviews 13:263-303.

GLASER R, SAWKa MN, BRUNE MF, WILDE SW 1980 Physiological responses to maximal effort on wheelchair and on armcrank ergometry. Fournal of Applied Physiology 48(6): 1060-1064.

HANSEN OE, MAGGIO M 1960 Static work and heart rate. International fournal of Angew Physiology 18:242-248.

HJELTNES N 1977 Oxygen uptake and cardiac output in graded arm exercise in paraplegics with low level spinal lesions. Scandinavian fournal of Rehabilitation Medicine 9:107-113. 
HoFFMAN MD 1986 Cardiorespiratory fitness and training in quadriplegics and paraplegics. Sports Medicine 3:312-330.

HoRvat M, FRENCH R, HensChen KA 1986 Comparison of the physiological characteristics of male and female able-bodied and wheelchair athletes. Paraplegia 24:115-22.

KAVANAGH T, ShePhaRD RJ 1973 The application of exercise testing to the elderly amputees. Canadian Medical Association fournal 108:314-317.

Kofsky PR, DAvis GM, ShePhaRd RJ, KEENE GC 1983 Field testing: Assessment of physical fitness of disabled adults. European fournal of Applied Physiology 51:109-120.

LUNDBERG $\AA 1980$ Wheelchair driving. Scandinavian fournal of Rehabilitation Medicine 12:67-72.

MCCANN BC 1984 Classification of the locomotor disabled for competitive sports: theory and practice. International fournal of Sports Medicine 5:167-170.

MOSTARDI RA, GANDEE RN 1981 Exercise training using arms and legs versus legs alone. Archives of Physical Medicine and Rehabilitation 62:334-336.

NAGLE FJ, RICHIE JP, GIESE MD 1984 VO2max responses in separate and combined arm and leg airbraked ergometer exercise. Medical Science and Sports Exercise 16(6):563-566.

Pandolf KB, Billing DS, Drolet LL, Pimental NA, Sawka MN 1984 Differentiated ratings of perceived exertion and various physiological responses during prolonged upper and lower body exercise. European fournal of Applied Exercise 53:5-11.

SAWKa MN, Miles DS, PETrowSKY JS 1982 Ventilation and acid-base equilibrium for upper body and lower body exercise. Aviation Space and Environmental Medicine 53(4):354-359.

Sawka MN, Foley ME, Pimental NA, Toner MM, Pandolf KB 1983 Determination of maximal aerobic power during upper-body exercise. Fournal of Applied Physiology: Environmental Exercise Physiology 54(1):113-117.

SAWKa MN, GlaSer RM, Wilde SW, Von LuhrTe TC 1980 Metabolic and circulatory responses to wheelchair and arm crank exercise. Fournal of Applied Physiology: Environmental Exercise Physiology 49(5):784-788.

SAWKa MN 1986 Physiology of upper body exercise. Exercise and Sport Science Review 14:175-211.

SHEPHARD RJ 1988 Sports medicine and the wheelchair athlete. Sports Medicine 5(4):226-247.

Smith PA, Glaser RM, Petrofsky JS 1983 Arm crank vs handrim wheelchair propulsion: Metabolic and cardiopulmonary responses. Archives of Physical Medicine and Rehabilitation 64(6):249-254.

WAHREN J, BYGDEMAN S 1971 Onset of angina pectoris in relation to circulatory adaptation during arm and leg exercise. Circulation 44:432-41.

WICKS JR, OLDRIDGE NB, CAMERON BJ, JONES NL 1983 Arm cranking and wheelchair ergometry in elite spinal cord-injured athletes. Medical Science in Sports and Exercise 15(3):224-231.

WICKS JR, LymbURNER K, Dindale SM, JONES NL 1978 The use of multistage exercise testing with wheelchair ergometry and arm cranking in subjects with spinal cord lesions. Paraplegia 15:252-261.

WILDE W, Miles DS, DURbin RJ, SAWKa MN, GLASER RM 1981 Evaluation of myocardial performance during wheelchair ergometer exercise. American fournal of Physical Medicine 60(6):277-290.

William J, Cottrell E, Powers SK, McKnight T 1983 Arm ergometry: a review of published protocols and the introduction of a new weight protocol. Fournal of Sports Medicine 23:107-112. 